Autorenkollektiv

Organic Chemistry 

Autorenkollektiv

\section{ORGANIC CHEMISTRY}

With 12 Figures and 59 Tables

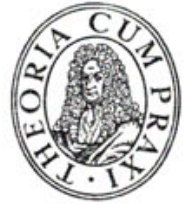

AKADEMIE-VERLAG · BERLIN 1982 
Die Originalausgabe erscheint im Springer-Verlag Berlin $\cdot$ Heidelberg $\cdot$ New York

Vertrieb ausschließlich für die DDR und die sozialistischen Länder

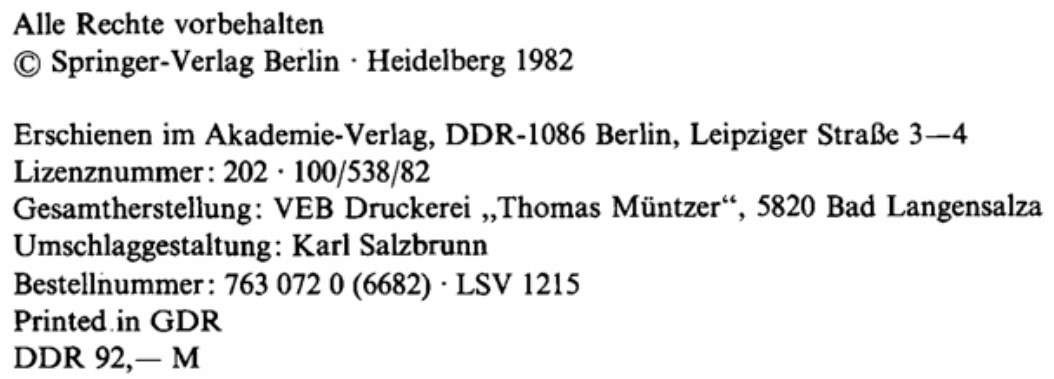


Managing Editor:

Dr. Friedrich L. Boschke

Springer-Verlag, Postfach 105280, D-6900 Heidelberg 1

Editorial Board:

Prof. Dr. Michael J. S. Dewar Department of Chemistry, The University of Texas Austin, TX 78712, USA

Prof. Dr. Jack D. Dunitz

Laboratorium für Organische Chemie der Eidgenössischen Hochschule Universitätsstraße 6/8, CH-8006 Zürich

Prof. Dr. Klaus Hafner Institut für Organische Chemie der TH Petersenstraße 15, D-6100 Darmstadt

Prof. Dr. Edgar Heilbronner Physikalisch-Chemisches Institut der Universităt Klingelbergstra $\mathrm{Be} 80, \mathrm{CH}-4000$ Basel

Prof. Dr. Shô Itô

Department of Chemistry, Tohoku University, Sendai, Japan 980

Prof. Dr. Jean-Marie Lehn Institut de Chimie, Université de Strasbourg, 1, rue Blaise Pascal, B. P. Z 296/R8, F-67008 Strasbourg-Cedex

Prof. Dr. Kurt Niedenzu University of Kentucky, College of Arts and Sciences Department of Chemistry, Lexington, KY 40506, USA

Prof. Kenneth N. Raymond Department of Chemistry. University of California. Berkeley, California 94720. USA

Prof. Dr. Charles W. Rees Hofmann Professor of Organic Chemistry, Department of Chemistry, Imperial College of Science and Technology, South Kensington, London SW7 2AY, England

Prof. Dr. Klaus Schäfer Institut für Physikalische Chemie der Universität Im Neuenheimer Feld 253, D-6900 Heidelberg 1

Prof. Dr. Georg Wittig Institut für Organische Chemie der Universität Im Neuenheimer Feld 270, D-6900 Heidelberg 1 
